\title{
Respiratory burst of intestinal macrophages in inflammatory bowel disease is mainly caused by $\mathrm{CD} 14^{+} \mathrm{L}^{+}$monocyte derived cells
}

\author{
J Rugtveit, G Haraldsen, A K Høgåsen, A Bakka, P Brandtzaeg, H Scott
}

\begin{abstract}
Macrophages play a crucial role in intestinal mucosal defence, forming dense subepithelial aggregates, particularly in the colon. One of their important bactericidal mechanisms is production of oxygen radicals but this may damage the intestinal epithelium, perhaps as an early step in inflammatory bowel disease (IBD). The potential for release of oxygen radicals from mucosal macrophages in IBD was measured and whether a difference exists between newly arrived $\left(\mathrm{CD14}^{+} \mathrm{L1}^{+}\right)$ monoctye-like cells and resident macrophages $\left(\mathrm{CD}_{14}^{-} \mathrm{L1}^{-}\right)$, without or with additional priming in vitro, was investigated. Lamina propria mononuclear cells from six patients with IBD and five with a normal intestine were isolated with an ethylenediaminetetra acetic acid/collagenase/dispase technique and cultured for three days. The cells were tested with or without interferon $\gamma(200 \mathrm{U} / \mathrm{ml})$ priming in the presence or absence of lipopolysaccharide $(1 \mu \mathrm{g} / \mathrm{ml})$ for the last 48 hours in culture. Samples from inflamed IBD mucosa depleted of $\mathrm{CD}^{+}$cells by immunomagnetic beads were compared with their undepleted counterparts and with samples from virtually normal mucosa from the same patients. The production of oxygen radicals was measured as the amount of reduced cytochrome $C$ $2 \cdot 5$ hours after triggering with phorbol 12myristate 13-acetate. The oxygen radical production in macrophages from moderately or severely inflamed mucosa was reduced by median $69 \%$ (range $22 \%-79 \%$, $\mathbf{p}<0.027)$ after depletion of $\mathrm{CD}^{+} 4^{+}$cells, reaching a level similar to that found for virtually normal samples from the same IBD patients. Furthermore, this production did not increase significantly in mucosal macrophages from normal reference mucosa and from virtually normal or inflamed IBD mucosa after priming with interferon $\gamma$ with or without addition of lipopolysaccharide. Upregulation of a respiratory burst in subepithelial resident macrophages is not a likely pathogenetic step in IBD. The increased oxygen radical production shown by macrophages from IBD lesions can, however, be ascribed to recently extravasated $\mathrm{CD}^{4} 4^{+} \mathrm{L1}^{+}$monocyte-like cells. Inhibition of extravasation of these reactive cells may form part of a therapeutic approach in the future.

(Gut 1995; 37: 367-373)
\end{abstract}

Keywords: inflammatory bowel disease, macrophages, respiratory burst, reactive oxygen species, CD14 antigen, $\mathrm{L} 1$ antigen.

Mucosal phagocytes are presumed to play an important role in local defence against invading micro-organisms. One of their major microbicidal mechanisms is 'respiratory burst', a term used for the production of oxygen intermediates such as superoxide, hydrogen peroxide, and hydroxyl groups, collectively called reactive oxygen intermediates (ROI). ${ }^{1} \mathrm{~A}$ potential side effect of ROI is cytotoxicity, which may cause local tissue damage. The dense subepithelial accumulation of macrophages in the intestinal mucosa ${ }^{2} 3$ suggests that activation of these cells with induction of respiratory burst and subsequent epithelial destruction may be an early step in the pathogenesis of inflammatory bowel disease (IBD). Macrophages newly recruited from peripheral blood $^{4}$ could also contribute to this local development. Previous investigations have reported increased levels of ROI production in mononuclear cells isolated from IBD mucosa, but a distinction has not been made between the macrophages with a monocyte-like phenotype and resident macrophages. ${ }^{5}$

We examined the respiratory burst of macrophages isolated from moderately or severely inflamed IBD mucosa compared with parallel samples obtained from virtually normal mucosa from the same patients. The effect of depleting the former cultures of recently recruited macrophages, defined by their expression of $\mathrm{CD} 14^{67}$ and the $\mathrm{L} 1$ antigen or calprotectin, ${ }^{4}$ was also studied. We also examined the responsiveness of mucosal macrophages to interferon $\gamma(\mathrm{IFN}-\gamma)$ and lipopolysaccharide (LPS) with regard to induction of respiratory burst, using in vitro matured monocytes as a positive control. Our results showed that the $\mathrm{CD} 14^{+} \mathrm{Ll}^{+}$subset was responsible for most of the increased respiratory burst activity of isolated macrophages from inflamed IBD mucosa, and that this activity could not be upregulated in resident macrophages by IFN- $\gamma$ and LPS in contrast to in vitro matured monocytes.

\section{Methods}

TISSUE SPECIMENS

The material included surgical specimens from six patients with IBD (one specimen from the ileum and five from the large bowel) 
and normal intestinal reference specimens (jejunum) from five organ donors in whom peripheral circulation was artificially maintained. Three of the IBD patients had Crohn's disease and three ulcerative colitis as judged by ordinary clinical and histopathological criteria. From each IBD patient specimens were taken from mucosa with macroscopically little or no inflammation (internal controls) as well as from mucosa with moderate or severe inflammation. The intensity of inflammation was further evaluated by conventional histological staining of paraffin sections from ethanol-fixed specimens ${ }^{8}$ taken from the respective mucosal areas. Microscopically, the inflammatory reaction was scored as 'no', 'mild', 'moderate', or 'severe', with special emphasis on the degree of mononuclear cell infiltration, crypt abscesses, and crypt destruction. Mucosa from the same patients showing no or only mild inflammation is referred to as 'histologically normal IBD mucosa'.

Two of the IBD patients were not treated with steroids at all and none received more than $15 \mathrm{mg}$ prednisolone per day during the last two weeks before surgery. Two patients were given mesalazine and one sulphazalasin in addition to prednisolone. Only one of the organ donors had received steroids for terminal brain oedema.

\section{MONOCYTES}

Monocytes from six healthy blood donors were isolated by Isopaque-Ficoll (Lymphoprep, Nycomed AS, Oslo, Norway) gradient centrifugation followed by plastic adherence in microtitre trays (Multiwell, Falcon, Becton Dickinson, UK) or to chamber slides (LABTEK, Nunc, Roskilde, DK); $3 \times 10^{6}$ mononuclear cells/ml in RPMI-1640 (Biowhittaker, Walkersville, MD) containing $5 \%$ fetal calf serum (Gibco BRL, Paisley, Scotland) were incubated for 1 hour followed by three gentle washings with prewarmed medium.

LAMINA PROPRIA MONONUCLEAR CELLS

Lamina propria mononuclear cells (LPMNC) were isolated by a modification of the technique described by Bull and Bookman. ${ }^{9}$ Briefly, the surgical specimens were washed extensively in calcium-free and magnesiumfree phosphate buffered saline (CMF-PBS), $\mathrm{pH} 7 \cdot 4$, before incubation in CMF-PBS with 1 mM dithiothreitol (DTT; Sigma Chemical Company, St Louis, MO) for 15 minutes at $20^{\circ} \mathrm{C}$ with shaking, followed by four incubations at $37^{\circ} \mathrm{C}$ in CMF-PBS with $5 \mathrm{mM}$ EDTA, 30 minutes each, also with shaking. After this procedure to remove epithelial cells, the mucosa with adjoining submucosa was dissected free from the muscularis propria, cut in small pieces, and incubated for 1-2 hours at $37 . \mathrm{C}$ in $25 \mathrm{mM}$ Hepes buffered RPMI-1640 with $1 \mathrm{mg} / \mathrm{ml}$ collagenase A from Clostridium perfringens (Boehringer Mannheim Biochemica, D-6800 Mannheim, Germany) $2 \mathrm{mg} /$ $\mathrm{ml}$ dispase (Boehringer), and $0.04 \mathrm{mg} / \mathrm{ml}$ DNase (Boehringer). The cell suspension was then filtered through a $200 \mu \mathrm{m}$ steel mesh (Bellco Glass Inc, Vineland, NJ) before Isopaque-Ficoll separation. The viability of the cells obtained was determined by fluorescence microscopy after staining with acridine orange/ethidium bromide. ${ }^{10}$

While keeping the LPMNC suspension at $4^{\circ} \mathrm{C}$, aliquots representing moderately or severely inflamed IBD mucosa were depleted of $\mathrm{CD}_{14}{ }^{+}$cells by immunomagnetic beads armed with antibodies to this antigen (Dynal, Oslo, Norway) and next cultured in parallel to undepleted counterparts as well as samples representing histologically normal mucosa from the same patients. The cells were seeded at a concentration of $3 \times 10^{6}$ cells $/ \mathrm{ml}$ in microtitre trays and on chamber slides coated with $0 \cdot 1 \%$ gelatin (Sigma), and $50 \%$ $(\mathrm{v} / \mathrm{v})$ inactivated human plasma, ${ }^{11-13}$ before adherent cells were obtained by washing away the non-adherent cells after 2-3 hours incubation as described above for blood monocytes.

\section{CELL CULTURES}

RPMI-1640 (Biowhittaker) was used as medium in all cultures, with the addition of $10 \%(\mathrm{v} / \mathrm{v})$ human serum (Sigma), penicillin $(100 \mathrm{U} / \mathrm{ml})$, streptomycin $(100 \mathrm{mg} / \mathrm{ml})$, gentamycin $(40 \mathrm{mg} / \mathrm{ml})$, amphotericin B $(0.25$ $\mathrm{mg} / \mathrm{ml})$, and L-glutamine $(2 \mathrm{mmol} / \mathrm{l})$.

Adherent LPMNC were cultured for 3 days with the addition of $1000 \mathrm{U} / \mathrm{ml}$ granulocyte/macrophage colony-stimulating factor (GM-CSF; kindly provided by ScheringPlough Research, Bloomfield, NJ) to increase the viability and survival of the cells ${ }^{14}$; in some experiments (see below) the last 2 days adding IFN- $\gamma$ at $200 \mathrm{U} / \mathrm{ml}$ (Genzyme Corp, Cambridge, MA), LPS at $1 \mu \mathrm{g} / \mathrm{ml}$ (from Escherichia coli strain-26 colon B-6, Sigma), or both at the same concentrations. In three experiments (one normal reference sample and two inflamed IBD specimens) the nonadherent LPMNC were cultured in parallel for comparison.

Adherent mononuclear cells from peripheral blood were cultured for 10 days, the last 2 days including parallels with addition of IFN- $\gamma(200$ $\mathrm{U} / \mathrm{ml}$ ) in the presence or absence of either LPS $(1 \mu \mathrm{g} / \mathrm{ml})$ or $\mathrm{GM}-\mathrm{CSF}(1000 \mathrm{U} / \mathrm{ml})$, or with all three agents combined at the concentrations mentioned. These experiments were performed to compare the responsiveness to stimulation with that observed for the adherent LPMNC cultures.

CELL NUMBERS AND PHENOTYPES IN CULTURES Determination of the cell number in the microtitre trays was performed by a modification of the method described by Gillies et al. ${ }^{15}$ Briefly, after measurement of the superoxide production the trays were centrifuged at $500 \mathrm{~g}$ for 3 minutes and emptied. The cells were air dried and fixed in $70 \%$ ethanol, stained with crystal violet, and solubilised; the relative cell number per well was determined spectrophotometrically. Standard curves constructed for monocytes and for adherent LPMNC were 
made and showed good correlation between changes in optical density (OD) and cell numbers $(r>0.9)$ within each tray, in accordance with previous reports. ${ }^{15}$ All the three categories of adherent LPMNC obtained from each IBD patient (see above) were examined in the same microtitre tray.

The percentage of macrophages in the three sample categories of adherent LPMNC from IBD patients was compared by applying butyrate esterase staining of acetone-fixed chamber slide cultures treated identically with those in the microtitre trays. To control for contamination of granulocytes, parallel preparations were stained by nuclear staining and also for chloroacetate esterase (neutrophilic granulocytes).

Immediately after separation by immunomagnetic beads, samples of CD14 ${ }^{+}$LPMNC from inflamed mucosa from four of the patients were fixed for 1 hour in $10 \%$ formalin and cytospins were prepared and incubated for 1 hour with tetramethylrhodamine isothiocyanate (TRITC) labelled rabbit IgG $(0.035 \mathrm{~g} / \mathrm{l})$ specific for the myelomonocytic marker L1 or calprotectin. ${ }^{16}$ TRITC labelled normal rabbit IgG at the same concentration was used as a negative control, and both conjugates had been prepared in our laboratory with a similar fluorochrome:protein ratio. ${ }^{17}$

To evaluate the efficiency of the depletion of the $\mathrm{CD} 14^{+}$LPMNC by immunomagnetic beads, acetone fixed cytospins of depleted samples were stained for CD14 by monoclonal antibody (mAb) against C14 (Becton Dickinson, Immunocytometry systems, 2350 Qume Drive, San Jose, CA 95131-1807) applied at $1 / 40$ for 1 hour followed by biotinylated horse anti-mouse IgG $(0.025 \mathrm{~g} / \mathrm{l}$; Vector Laboratories, CA, USA) for 1.5 hours and streptavidin-Texas red (Bethesda Research Laboratories, MD, USA) at $1 / 400$ for 30 minutes. Negative controls were obtained by omitting the primary antibody.

The maturation of the monocyte cultures was evaluated after one week by examining the expression of differentiation antigens defined by mAbs of the Max series (MAX 1, MAX 3, and MAX 11$)^{18}$ in a cell ELISA performed as described previously. ${ }^{19}$

\section{ANALYSIS OF SUPEROXIDE PRODUCTION}

The production of superoxide anions was measured in a rapid spectrophotometric microassay $^{20}$ as the amount of reduced cytochrome $\mathrm{C}$ expressed by the proportional increase of $O D$ at $550 \mathrm{~nm}$ during the 2.5 hours after addition of a reactant solution. Briefly, after centrifugation of the microtitre trays ( 3 minutes, $300 \mathrm{~g}$ ), aspiration of the cell culture medium, and gentle washing with prewarmed colourless RPMI-1640 (Gibco, Life Technologies, Paisley, Scotland), a mixture of 2 $\mathrm{mg} / \mathrm{ml}$ cytochrome C (Sigma) and $200 \mathrm{nM}$ phorbol 12-myristate 13-acetate (PMA, Sigma) in $100 \mu$ l colourless RPMI-1640 was added to each well and incubated for 2.5 hours at $37^{\circ} \mathrm{C}$. Superoxide dismutase (SOD, Sigma) was added at $300 \mathrm{U} / \mathrm{ml}$ to selected wells to block the superoxide anion-dependent reduction of cytochrome $C$; these wells were used as blanks. In four of the experiments, freshly isolated monocytes were analysed in the same microtitre tray as the adherent LPMNC; these wells were used as positive controls. The results were recorded in an automated enzyme immunoassay reader (Termomax Microplate Reader; Molecular Devices Corp, Mento Park, CA) connected to a Macintosh computer with a microtitre tray reader program (Softmax program for Macintosh, version 2.2, Molecular Devices, Menlo Park, CA).

\section{EFFECT OF ISOLATION PROCEDURE}

The LPS concentration in the enzyme solution after 1 hour's treatment of the mucosal tissue was tested by the chromogenic limulus amebocyte lysate assay (Kabi Vitrum AB, Stockholm, Sweden) and found to be in the range of $0.5-8.5 \mathrm{ng} / \mathrm{ml}$. To evaluate the possible influence of pre-exposure to collagenase $A$ and dispase or endotoxin during the isolation procedure on the superoxide production and its response to priming with IFN- $\gamma$ in the LPMNC, monocyte derived macrophages were used as a model system. Adherent blood mononuclear cells cultured for 8 days were subjected to either collagenase A $(1 \mathrm{mg} / \mathrm{ml})$ and dispase $(2 \mathrm{mg} / \mathrm{ml})$ of LPS $(10 \mathrm{ng} / \mathrm{ml})$ for 1 hour, centrifuged briefly ( $300 \mathrm{~g}, 3$ minutes) to avoid loss of non-adherent cells, washed gently with prewarmed RPMI-1640, and then (parallel to the adherent LPMNC) cultured for 3 days with GM-CSF $(1000 \mathrm{U} / \mathrm{ml})$, the last 2 days with or without IFN- $\gamma(200 \mathrm{U} / \mathrm{ml})$.

\section{STATISTICS}

The data were considered non-parametric and comparisons between groups were performed by the Mann-Whitney $U$ test for unpaired samples (two tailed) and by the Wilcoxon signed rank test for paired samples.

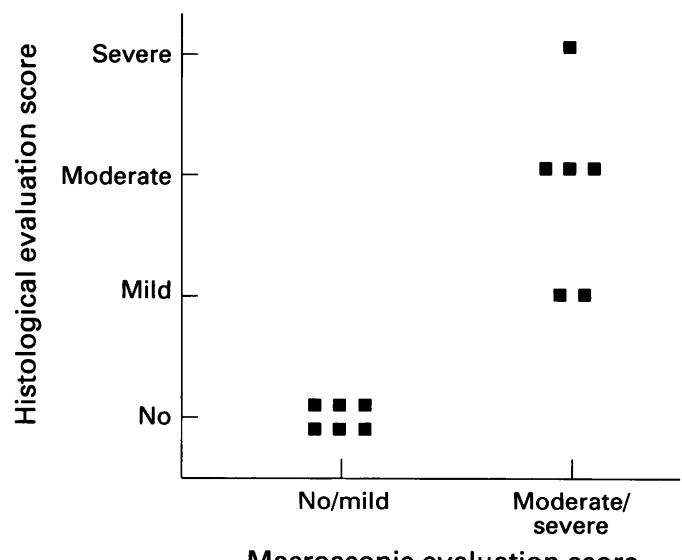

Macroscopic evaluation score

Figure 1: Scatter plot comparing degree of inflammation as judged macroscopically and by conventional

histopathological examination of mucosal inflammatory bowel disease specimens. One specimen with no or mild macroscopic inflammation and one with moderate to severe inflammation were included from each patient (specimens with histological score of 'no' or only 'mild' inflammation are referred to as 'histologically normal' inflammatory bowel disease mucosa in the text). 


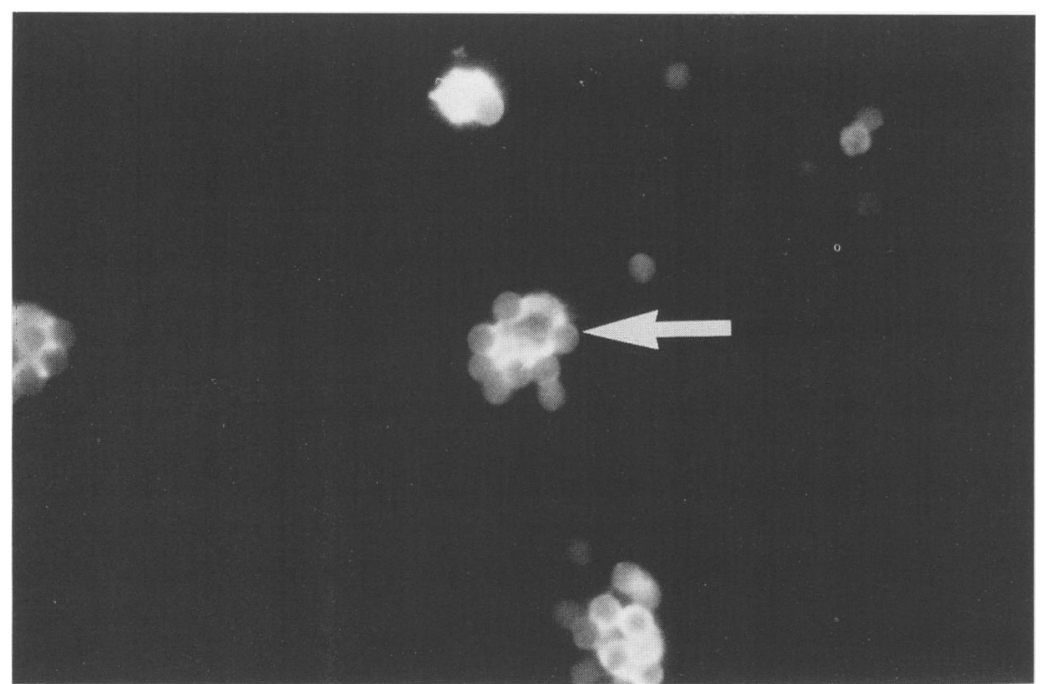

Figure 2: Coexpression of L1 and CD14 in isolated lamina propria mononuclear cells from inflamed inflammatory bowel disease mucosa. Immunofluorescence microscopy showing the presence of L1 in cells isolated by immunomagnetic beads armed with antibody to CD14 (arrow indicates L1-positive cell surrounded by beads).

\section{Results}

\section{HISTOLOGICAL EVALUATION OF DEGREE OF} INFLAMMATION

The histopathological findings in intestinal tissue sections taken from the IBD specimens confirmed the macroscopical evaluation of the mucosal samples (Fig 1). This comparison was performed in a blinded manner by an experienced histopathologist.

VIABILITY AND CELL PHENOTYPES IN CULTURES The viability of LPMNC after density gradient centrifugation was median $92 \%$ (range $85 \%$ $96 \%$ ); after adherence separation all cells were judged to be viable. The percentage of mononuclear cells decorated with immunomagnetic beads determined microscopically in conjunction with the depletion of $\mathrm{CD} 4^{+}$cells, was median 3\% (range 1\%-5\%). Staining for CD14 in LPMNC after depletion showed that $>80 \%$ of the $\mathrm{CD}^{+} 4^{+}$cells were removed by the immunomagnetic beads. The percentage of macrophages determined by butyrate esterase staining in adherent LPMNC (counted immediately after adherence separation) from histologically normal IBD mucosa (median $16 \cdot 0 \%$, range $15 \cdot 7 \%-17 \cdot 8 \%$ ) was not significantly different from that in samples representing mucosa with moderate or severe inflammation (median $17 \cdot 6 \%$, range $15.3 \%$ $20 \cdot 4 \%$ ). However, the proportion of such macrophages in the CD14 depleted counterparts of the latter samples (median $12.8 \%$,

TABLE I Effect of isolation procedure on superoxide production in monocyte derived macrophages

\begin{tabular}{lll}
\hline & \multicolumn{2}{l}{ Priming with IFN- $\gamma(48 \mathrm{~h})$} \\
\cline { 2 - 3 } Pre-exposure & None & $200 \mathrm{U} / \mathrm{ml}$ \\
\hline Control & $0.02(0-0.04) \dagger$ & $0.08(0.05-0 \cdot 16)$ \\
Lipopolysaccharide $(10 \mathrm{ng} / \mathrm{ml})$ & $0.03(0.01-0.04)$ & $0 \cdot 10(0.07-0 \cdot 14)$ \\
Collagenase $(1 \mathrm{mg} / \mathrm{ml})$ and dispase $(2 \mathrm{mg} / \mathrm{ml})$ & $0.05(0.02-0 \cdot 12)$ & $0.12(0.06-0 \cdot 14)$ \\
\hline
\end{tabular}

${ }^{\star}$ Exposure $(1 \mathrm{~h})$ to lipopolysaccharide or collagenase $\mathrm{A}$ and dispase 3 days before testing (three blood donors, same experiment). + Values expressed as change in optical density at $550 \mathrm{~nm}$ and adjusted to $50000 \mathrm{cell} /$ well (median and observed ranges); detection limit: 0.005 . range $12 \cdot 1 \%-15 \cdot 1 \%$ ) was significantly reduced $(\mathrm{p}=0.026)$ although the difference was relatively small $(27 \%$ reduction).

Median $70 \%$ (range $65 \%-77 \%$ ) of the cells isolated by the anti-CD14 immunomagnetic beads were positive for the myelomonocytic L1 marker (Fig 2). After adherence separation the fraction of granulocytes in the cultures was median $1.0 \%$ (range $0.5 \%-4.0 \%$ ), and at the time of the superoxide analysis this contamination was reduced to $<0.5 \%$ as determined by nuclear morphology (routine staining) and chloroacetate esterase staining. The fraction of monocytes in the monocyte cultures after 8 days was median $93 \%(90 \%-95 \%)$ as determined by butyrate esterase staining and no granulocytes were seen.

\section{SUPEROXIDE PRODUCTION}

The PMA stimulated superoxide production in monocyte derived macrophages, both with or without IFN- $\gamma$ priming for 2 days, was not inhibited by incubation of the cultures for 1 hour with medium in the presence of LPS $(10 \mathrm{ng} / \mathrm{ml})$ or collagenase $\mathrm{A}(1 \mathrm{mg} / \mathrm{ml})$ and dispase $(2 \mathrm{mg} / \mathrm{ml}) 3$ days before testing (Table I). Also, the addition of LPS $(1 \mu \mathrm{g} / \mathrm{ml})$ or GM-CSF ( $1000 \mathrm{U} / \mathrm{ml})$ together with IFN- $\gamma$ for 48 hours did not affect the increase in respiratory burst compared with addition of IFN- $\gamma$ alone (Table II). Nor did GM-CSF $(1000 \mathrm{U} / \mathrm{ml})$ alone increase the respiratory burst in monocyte derived macrophages $(n=3$, data not shown) compared with controls.

The superoxide production in the undepleted LPMC cultures from moderately or severely inflamed IBD mucosa was significantly increased $(p=0.028)$ by median $30 \%$ (range 20\%-200\%) compared with samples representing histologically normal mucosa from the same patients; after CD14 depletion this level was significantly $(\mathrm{p}=0.027)$ reduced by median $69 \%$ (range $22 \%-79 \%$ ), and became similar to that of the undepleted samples from histologically normal mucosa of the same patients (Fig 3).

There was no significant increase in PMA stimulated superoxide production in any of the samples of adherent LPMNC from IBD patients after 2 days with IFN- $\gamma(200 \mathrm{U} / \mathrm{ml})$ and LPS $(1 \mu \mathrm{g} / \mathrm{ml})$. In adherent LPMNC from normal reference mucosa a small increase was observed (median 30\%, range 0\%-120\%) after priming with LPS with or without IFN- $\gamma$ (Fig 4) but this trend did not reach statistical significance. By contrast, there was a significant increase (median $107 \%$, range $30 \%-$ $300 \% ; \mathrm{p}=0.028)$ in the superoxide production of monocytes cultured for 8 days and then subjected to the same priming (Table II), and also when the monocytes had been pre-exposed to collagenase and dispase or LPS as mentioned above (data not shown). These monocytes had reached a mature phenotype as determined by cell ELISA for the antigens defined by the mAbs of the MAX series (Table III). The superoxide production per macrophage (butyrate esterase positive cell) in nonadherent LPMNC was in each case less than 
TABLE II Superoxide production in monocyte cultures

\begin{tabular}{ll}
\hline Stimulation (48 $h$ from day 8$)$ & $\begin{array}{l}\text { Change in OD at } 550 \mathrm{~nm} \\
\left(\text { median }(\text { range) })^{\star}\right.\end{array}$ \\
\hline Control (day 1) freshly isolated $(\mathrm{n}=6)$ & $0 \cdot 22(0 \cdot 16-0 \cdot 31) \dagger$ \\
Control (day 10) only medium from day $8(\mathrm{n}=6)$ & $0 \cdot 07(0 \cdot 02-0 \cdot 10)$ \\
IFN- $\gamma(200 \mathrm{U} / \mathrm{ml})(\mathrm{n}=6)$ & $0 \cdot 16(0 \cdot 12-0 \cdot 21)$ \\
IFN- $\gamma(200 \mathrm{U} / \mathrm{ml})+\mathrm{LPS}(1 \mu \mathrm{g} / \mathrm{ml})(\mathrm{n}=6)$ & $0 \cdot 15(0 \cdot 11-0 \cdot 18)$ \\
IFN- $\gamma(200 \mathrm{U} / \mathrm{ml})+\mathrm{GM}-\mathrm{CSF}(1000 \mathrm{U} / \mathrm{ml})(\mathrm{n}=3)$ & $0 \cdot 15(0 \cdot 11-0 \cdot 18)$ \\
IFN- $\gamma(200 \mathrm{U} / \mathrm{ml})+\mathrm{GM}-\mathrm{CSF}(1000 \mathrm{U} / \mathrm{ml})+\mathrm{LPS}(1 \mu \mathrm{g} / \mathrm{ml})(\mathrm{n}=3)$ & $0 \cdot 17(0 \cdot 16-0 \cdot 18)$
\end{tabular}

$\mathrm{OD}=$ optical density; IFN- $\gamma=$ interferon $\gamma$; LPS=lipopolysaccharide; GM-CSF=granulocyte

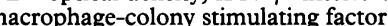

${ }^{\star}$ Results adjusted to 50000 cells/well. $†$ Detection limit: OD 0.005 .

one third of that in the adherent cells from the same patient $(p<0.05)$, as determined in two inflamed IBD specimens and in one normal reference sample (data not shown).

\section{Discussion}

Functional properties of macrophages obtained from IBD mucosa were evaluated in relation to phenotype by studying their inherent and inducible capacity for respiratory burst (release of ROI). In the isolation procedure, LPMNC were exposed to enzymes (collagenase and dispase) and trace amounts of LPS, which might have interfered with their function. The LPS concentration in the supernatant after enzymatic tissue digestion for 1 hour was $0.5-8.5 \mathrm{ng} / \mathrm{ml}$. Incubation for 1 hour with trace amounts of LPS ( $1 \mathrm{ng} / \mathrm{ml}$ ) has been reported to block the priming effect of IFN- $\gamma$ on the release of ROI from murine peritoneal macrophages. ${ }^{21}$ Furthermore, the addition of $10 \mathrm{ng} / \mathrm{ml}$ of LPS together with IFN- $\gamma$

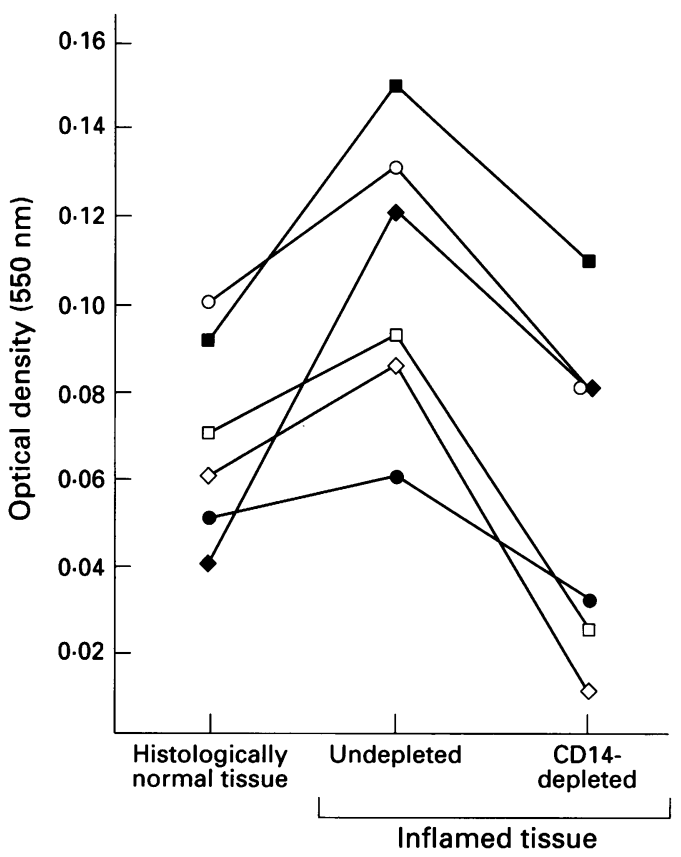

Figure 3: Superoxide production in adherent lamina propria mononuclear cells from inflammatory bowel disease tissue expressed as the proportional changes in optical density due to a reduction of cytochrome $C$ in the reacting solution added to the culture $2 \cdot 5$ hours before measuring at $550 \mathrm{~nm}$. Interferon- $\gamma(I F N-\gamma)(200 \mathrm{U} / \mathrm{ml})$ and lipopolysaccharide (LPS) $(1 \mu \mathrm{g} / \mathrm{ml})$ were added for the last 48 hours of 3 day cultures. Parallel samples from the same patient are connected by lines (closed symbols, Crohn's disease; open symbols, ulcerative colitis). Results were adjusted to 50000 cells/well $(n=6)$. Granulocyte macrophage colony stimulating factor $(1000 \mathrm{U} / \mathrm{ml})$ was added to all media at the start of culture. Detection limit: optical density 0.005 .
$(0 \cdot 1-1000 \mathrm{U} / \mathrm{ml})$ for 48 hours to cultures of murine peritoneal macrophages reduced the IFN- $\gamma$ dependent upregulation of the ROI release. ${ }^{22}$ However, using in vitro matured monocytes as a macrophage model system, we did not find that LPS reduced the priming effect of IFN- $\gamma$ on ROI production, either when the cultures were pre-exposed to LPS at $10 \mathrm{ng} / \mathrm{ml}$ for 1 hour at the beginning of the culture period, or when LPS at relatively high concentrations $(1 \mu \mathrm{g} / \mathrm{ml})$ was incubated together with IFN- $\gamma$ for 48 hours.

Proteolytic enzymes have been reported to exert a priming effect on PMA triggered ROI production in murine peritoneal macrophages, ${ }^{23}$ out Mahida et $a l^{5}$ could not find such an effect on human monocytes. both studies measured ROI production directly after the enzyme treatment. In our study, in vitro matured monocytes showed no inhibition of PMA triggered ROI production or its priming by IFN- $\gamma$ measured 3 days after exposure to collagenase and dispase for 1 hour. Conversely, the PMA triggered ROI release tended to be increased, but not significantly so.

In our study, adherent LPMNC cultures were allowed to reconstitute for 24 hours in medium containing GM-CSF to improve the viability before priming with IFN- $\gamma$, with or without addition of LPS. Coleman et $a l^{24}$ found that GM-CSF stimulated the oxidative metabolism after 48 hours' incubation of murine resident peritoneal macrophages, but other studies did not show any effect of GMCSF on ROI production in human monocyte derived macrophages. ${ }^{25-27}$ Our results with in vitro matured monocytes showed no additional increased in PMA triggered ROI production

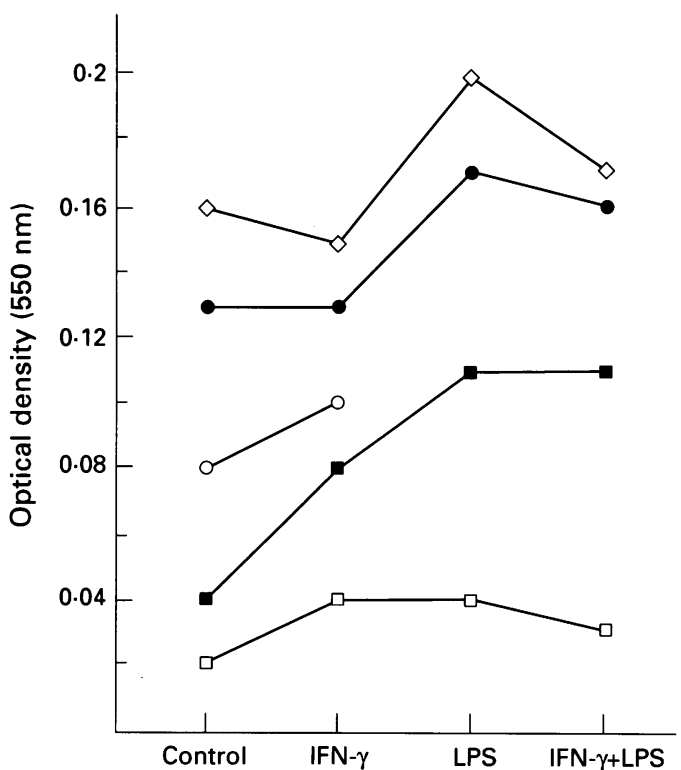

Figure 4: Superoxide production in adherent lamina propria mononuclear cells from normal reference mucosa expressed as the proportional changes in optical density due to a reduction of cytochrome $C$ in the reacting solution added to the cultures $2 \cdot 5$ hours before measuring at $550 \mathrm{~nm}$. Interferon- $\gamma(I F N-\gamma)(200 \mathrm{U} / \mathrm{ml})$, lipopolysaccharide (LPS) ( $\mu \mathrm{g} / \mathrm{ml})$, or both (same concentrations) were added for the last 48 hours of the 3 day culture. Results were adjusted to 50000 cells/well $(n=5)$. All media contained granulocyte macrophage colony stimulating factor $1000 \mathrm{U} / \mathrm{ml}$ at the start of culture. Detection limit: optical density 0.005 . 
TABLE III Expression of maturation antigens in monocyte cultures ${ }^{\star}$

\begin{tabular}{lll}
\hline & \multicolumn{2}{l}{$O D$ (median (range) $\dagger$} \\
\cline { 2 - 3 } Antibody & 1 Day culture & 7 Day culture \\
\hline MAX 1 & $0.01(0-0.07)$ & $0 \cdot 11(0.05-0.20)$ \\
MAX 3 & $0.02(0-0.02)$ & $0 \cdot 12(0.02-0.18)$ \\
MAX 11 & $0(0-0.04)$ & $0.095(0.05-0 \cdot 17)$ \\
\hline
\end{tabular}

^Expression detected by the mAbs of the MAX series measured in cell-ELISA (three donors, same experiment). +Values expressed as optical density at $550 \mathrm{~nm}$; detection limit: $0 \cdot 005$.

when GM-CSF was added to IFN- $\gamma$; nor was any increase noted when GM-CSF alone was added to the medium. On the basis of this macrophage model system, we concluded that the isolation procedure and culture conditions of lamina propria macrophages had no serious effects on the functional variable under investigation. Furthermore, the functional properties of LPMNC subjected to bead depletion of $\mathrm{CD}_{14}{ }^{+}$cells were apparently not adversely affected with respect to interleukin 6 production as determined in three IBD samples (unpublished observations).

No significant increase was observed in PMA triggered superoxide production by macrophages from IBD mucosa as well as from normal reference mucosa after priming with IFN- $\gamma$ for 2 days, with or without addition of LPS. This was in striking contrast to the results obtained with in vitro matured monocytes, also when these cells had been subjected to similar enzyme and LPS concentrations 3 days before analysis, like the intestinal macrophages. Our findings agreed with previous results reported for isolated human intestinal macrophages examined by semiquantitative methods. ${ }^{5} \mathrm{~A}$ similar unresponsiveness of murine Kupffer cells has also been reported. ${ }^{28}$

The unresponsiveness of mucosal macrophages to IFN- $\gamma$ in the presence or absence of LPS observed in the present test system is not a general phenomenon; similar conditions resulted in significantly increased production of IL-1 $\alpha$ (Rugtveit et al, personal communication). Steroids might have inhibited the priming by IFN- $\gamma$ for superoxide production, but such an effect has not been observed ${ }^{29}$ and only one of the organ donors had received this treatment.

We found a decrease in ROI production (median 69\%) when LPMNC isolated from inflamed mucosa were depleted of $\mathrm{CD} 14^{+}$ cells. This significant reduction documented for the first time that $\mathrm{CD} 14^{+}$cells are the main contributors to the increased ROI production shown by macrophages from inflamed IBD mucosa, as observed both previously ${ }^{5}$ and in this study. The strong increase in respiratory burst activity of in vitro matured monocytes after IFN- $\gamma$ priming with or without LPS, and the maintenance of such high activity in monocytes cultured for more than 1 week in the presence of IFN- $\gamma^{30}$ and LPS, ${ }^{31}$ supported the possibility that monocytes extravasating at inflammatory sites retain a high capacity for respiratory burst. Using the myelomonocytic L1 marker or calprotectin, ${ }^{32}$ we have previously shown a relative increase in the monocyte-like fraction of cells in IBD mucosa with increasing degree of inflammation. ${ }^{4}$ The high percentage of $\mathrm{CD} 14^{+}$cells which reacted with antibody to L1 in LPMNC isolated from inflamed IBD mucosa substantiated our conclusion that most $\mathrm{CD} 14^{+}$cells responsible for an increased respiratory burst have been recently extravasated in the lesion.

The difference in respiratory burst activity observed between adherent LPMNC from inflamed and histological normal IBD mucosa remained unchanged after stimulation with IFN- $\gamma$ in the presence or absence of LPS. This finding was compatible with the notion that inflamed IBD mucosa contains a fraction of already highly activated macrophages newly derived from peripheral blood, and that resident macrophages in the mucosa of IBD patients are resistant to an upregulation of respiratory burst by inflammatory mediators such as IFN- $\gamma$ and by LPS. The same was found to hold true for macrophages in LPMNC cultures obtained from normal reference mucosa. The significant response to the same agents seen in the in vitro-matured monocytes 3 days after incubation with LPS and enzymes, suggested that the absent upregulation in resident macrophages was not a result of the experimental conditions but reflected an intrinsic property of these cells. Their lack of response to LPS is also in line with the view that CD14 is crucial for the cellular recognition and stimulatory effect of this factor. ${ }^{33}$ It would be interesting to compare the results we obtained for intestinal resident macrophages with those of similar studies of such cells from other human mucosae as well as non-mucosal tissues. As far as we know there are no such data available.

In conclusion, we found an increased respiratory burst in isolated macrophages from mucosal areas with moderate or severe inflammation compared with virtually normal mucosa from the same IBD patients tested in parallel. Our results strongly suggested that this increased respiratory burst arose in recently recruited $\mathrm{CD}_{14}^{+} \mathrm{Ll}^{+}$monocyte derived cells rather than being explained by inflammation induced upregulation of such activity in resident macrophages. Inhibition of extravasation of the $\mathrm{C}_{14}{ }^{+} \mathrm{Ll}^{+}$subset might play a part in treatment in the future. Based on our findings, stimulation of respiratory burst in the normal subepithelial population of mucosal macrophages is not likely to be involved in the pathogenesis of IBD.

Grant support was received from The Research Council of Norway, The Norwegian Cancer Society, Medinova Governmental Research Organisation, and Familien Blix' Fond.

JR is a Research Fellow of the University of Oslo.

1 Babior BM. The respiratory burst of phagocytes. $f$ Clin Invest 1984; 73 (3): 599-601.

2 Hume DA, Allan W, Hogan PG, Doe WF. Immunohistochemical characterisation of macrophages in human liver and gastrointestinal tract: expression of CD4, HLA-DR, OKM1, and the mature macrophage marker
$25 \mathrm{~F} 9$ in normal and diseased tissue. $\mathcal{F}$ Leukoc Biol 1987;
$\mathbf{4 2}$ (5): 474-84.

3 Selby WS, Janossy G, Mason DY, Jewell DP. Expression of HLA-DR antigens by colonic epithelium in inflammatory bowel disease. Clin Exp Immunol 1983; 53 (3): 614-8. 
4 Rugtveit J, Brandtzaeg P, Halstensen T, Fausa O, Scott H. Increased macrophage subset in inflammatory bowe disease: apparent recruitment from peripheral blood monocytes. Gut 1994; 35: 669-74.

5 Mahida YR, Wu KC, Jewell DP. Respiratory burst activity of intestinal macrophages in normal and inflammatory bowel disease. Gut 1989; 30 (10): 1362-70.

6 Hogg N, MacDonald S, Slusarenko M, Beverley PC. Monoclonal antibodies specific for human monocytes, granulocytes and endothelium. Immunology 1984; 53 (4): 753-67.

7 Allison MC, Cornwall S, Poulter LW, Dhillon AP, Pounder RE. Macrophage heterogeneity in normal colonic mucosa and in inflammatory bowel disease. Gut 1988; 29 (11): 1531-8.

8 Brandtzaeg P. Mucosal and glandular distribution of immunoglobulin component. Immunohistochemistry with a cold ethanol-fixation technique. Immunology 1974 26: 1101-14.

9 Bull DM, Bookman MA. Isolation and functional characterization of human intestinal mucosal lymphoid cells. fClin Invest 1977; 59: 966-74.

10 Parks D, Bryan V, VT. O, Herzenberg L. Antigen specific identification and cloning of hybridomas with a fluorescence activated cell sorter. Proc Natl Acad Sci USA 1979; cence acti 1962 .

11 Mahida YR, Wu KC, Jewell DP. Characterization of antigen-presenting activity of intestinal mononuclear cells isolated from normal and inflammatory bowel disease colon and ileum. Immunology 1988; 65 (4): 543-9.

12 Lin HS, Gordon S. Secretion of plasminogen activator by bone marrow-derived mononuclear phagocytes and its enhancement by colony-stimulating factor 7 Exp Med $1979 ; 150$ (2): $231-45$.

13 Bevilacqua MP, Amrani D, Mosesson MW, Bianco C. Receptors for cold-insoluble globulin (plasma fibronectin) on human monocytes. $\mathcal{F}$ Exp Med 1981; 153 (1): 42-60.

14 Lopez M, Marinache C, Canepa S, Chokri M, Scotto F, Bartholeyns J. Autologous lymphocytes prevent the death of monocytes in culture and promote, as do GM-CSF IL- 3 and M-CSF, their differentiation into macrophages. f Immunol Methods 1993; 159: 29-38.

15 Gillies R, Didier N, Denton M. Determination of cell number in monolayer cultures. Anal Biochem 1986; 159: 109-13.

16 Dale I, Brandtzaeg P, Fagerhol MK, Scott H. Distribution of a new myelomonocytic antigen (L1) in human peripheral blood leukocytes. Immunofluorescence and immunoperoxidase staining features in comparison with lysozyme and lactoferrin. Am $\mathcal{f}$ Clin Pathol 1985; 84 (1): lysozyme.

17 Brandtzaeg P. Conjugates of immunoglobulin $G$ with different fluorochromes. I. Characterization by anionicexchange chromatography. Scand $\mathcal{f}$ Immunol 1973; 2 273-90.

18 Andreesen R, Mackensen A, Osterholz J, Brugger W, Lohr GW. Microculture assay for human macrophage maturation in vitro. Cell-ELISA analysis of differentiation antigen expression. Int Arch Allergy Appl Immunol 1988; 86 (3): $281-7$

19 Kvale D, Brandtzaeg P, Lovhaug D. Up-regulation of the expression of secretory component and HLA molecules in a human colonic cell line by tumour necrosis factor-alpha and gamma interferon. Scand $\mathcal{F}$ Immunol 1988; 28 (3): 351-7.

20 Pick E, Mizel D. Rapid microassays for the measuremen of superoxide and hydrogen peroxide production by macrophages in culture using an automatic enzyme immunoassay reader. F Immunol Methods 1981; 46 (2): 211-26.

21 Ding AH, Nathan CF. Trace levels of bacterial lipopolysaccharide prevent interferon-gamma or tumour necrosis factor-alpha from enhancing mouse peritoneal macrophage respiratory burst capacity. F Immunol 1987; macrophage resp

22 Murray HW, Spitalny GL, Nathan CF. Activation of mouse peritoneal macrophages in vitro and in vivo by interferongamma. F Immunol 1985; 134 (3): 1619-22.

23 Johnston RJ, Chadwick DA, Cohn ZA. Priming of macrophages for enhanced oxidative metabolism by exposure to proteolytic enzymes. $\mathcal{f}$ Exp Med $1981 ; 153$ (6): $1678-83$.

24 Coleman DL, Chodakewitz JA, Bartiss AH, Mellors JW. Granulocyte-macrophage-colony-stimulating factor enhances selective effector functions of tissue derived macrophages. Blood 1988; 72: 573-8.

25 Sullivan GW, Carper HT, Mandell GL. The effect of three human recombinant hematopoietic growth factors human recombinant hematopoietic growth factors
(granulocyte-macrophage colony-stimulating factor granulocyte colony-stimulating factor, and interleukin-3) on phagocyte oxidative activity. Blood 1993;81 (7) 1863-70.

26 Kharazmi A, Nielsen H, Hovgaard D, Borregaard N, Nissen NI. Modulation of neutrophil and monocyte function by recombinant human granulocyte macrophage colony-stimulating factor in patients with lymphoma. Eur f Clin Invest 1991; 21 (2): 219-24.

27 Nathan CF, Prendergast TJ, Wiebe ME, et al. Activation of human macrophages. Comparison of other cytokines with interferon-gamma. $f$ Exp Med 1984; 160 (2): 600-5.

28 Lepay DA, Nathan CF, Steinman RM, Murray HW, Cohn ZA. Murine Kupffer cells. Mononuclear phagocytes deficient in the generation of reactive oxygen intermediates. F Exp Med 1985; 161 (5): 1079-96.

29 Szefler SJ, Norton CE, Ball B, Gross JM, Aida Y, Pabst MJ. IFN-gamma and LPS overcome glucocorticoid inhibition of priming for superoxide release in human monocytes. Evidence that secretion of IL- 1 and tumor necrosis factoralpha is not essential for monocyte priming. F Immunol 1989; 142 (11): 3985-92.

30 Nathan CF, Murray HW, Wiebe ME, Rubin BY. Identification of interferon-gamma as the lymphokine that activates human macrophage oxidative metabolism and antimicrobial activity. F Exp Med 1983; 158 (3): 670-89.

31 Pabst MJ, Hedegaard HB, Johnston RJ. Cultured human monocytes require exposure to bacterial products to maintain an optimal oxygen radical response. $\mathcal{F}$ Immuno 1982; 128 (1): 123-8.

32 Brandtzaeg P, Dale I, Fagerhol MK. Distribution of a formalin-resistant myelomonocytic antigen (L1) in human tissues. I. Comparison with other leukocyte markers by paired immunofluorescence and immunoenzyme staining. Am f Clin Pathol 1987; 87 (6): 681-99.

33 Ulevitch RJ, Tobias PS. Recognition of endotoxin by cells leading to transmembrane signaling [Review]. Curr Opin Immunol 1994; 6 (1): 125-30. 